\title{
RECOMBINATION IN DROSOPHILA WILLISTONI
}

\author{
Z. M. FRANCA, A. BRITO DA CUNHA and M. C. GARRIDO \\ Departamento de Biologia Geral, Faculdade de Filosofia, Ciências e Letras da Universidade de Sao \\ Paulo, Caixa Postal 8105, Sao Paulo, Brasil
}

Received 26.vi.67

\section{Introduction}

KNOWLEDGE of Drosophila genetics was acquired mainly through work on Nearctic species. Conclusions reached from studies of these species are frequently extended to those of other regions. Biological generalisations are hazardous especially when they involve adaptive characters of organisms living under different conditions. This is especially true for genetic systems which, owing to their crucial adaptational importance, must be highly sensitive to natural selection. The comparative analysis of the genetic systems of related species living in different environments is necessary before generalisations can be made and this is one of the ways of understanding their adaptive meaning and evolutionary origin.

Recombinant chromatids produced by crossing-over within relatively inverted segments are eliminated. Data obtained in Nearctic species of Drosophila show that heterozygous inversions also suppress effective crossing over outside the inverted segments. This is well known for inversions like Payne and Curly of D. melanogaster and for inversions in D. pseudoobscura, $D$. persimilis, $D$. virilis and particularly of $D$. robusta (review in Carson, 1953). No recombination was found by Carson (1953) within or between two heterozygous inversions in opposite arms of the second chromosomes of $D$. robusta and separated by a distance corresponding to 37.6 per cent. of the chromosome length. Similar results were obtained for inversions in the $\mathrm{X}$ chromosome.

On the basis of results obtained with Nearctic species, it is generally believed that there is no crossing over at spermatogenesis in Drosophila. The absence of crossing over in $D$. melanogaster males has been well known since Morgan's work (1914), which has been confirmed by many authors. Patterson and Suche (1934), for example, found only one recombinant among 8329 third chromosomes marked from tip to tip with eight recessive genes.

Here we present new data on recombination between heterozygous inversions in males and females of $D$. willistoni, a Neotropical species characterised by an abundance of inversions in its natural populations (da Cunha et al., 1950, 1954, 1959).

\section{MAterial AND methods}

Two naturally occurring inversions of the second chromosome originally obtained from populations on the island of São João, Angra dos Reis were studied. The second chromosome of $D$. willistoni is metacentric. One of the inversions, $R e$, is in the right arm and is terminal. The other inversion, $L f$, is sub-terminal and in the left arm. Their alternative standard gene 
arrangements are $R E$ and $L F$. The centromere will be represented as a point between the indications of the gene arrangements. A standard chromosome will be RE.LF, while the inverted will be Re.Lf, RE.Lf or Re.LF.

Two homozygous strains were obtained: RE.LF/RE.LF and Re.Lf/Re.Lf. Hybrid males and females $R E . L F / R e . L f$ were produced and crossed with homozygous standard females or males, Re.Lf/Re.Lf. The analysis of the salivary gland chromosomes of the $F_{1}$ larvae of these crosses give the frequencies of the non-recombinant, $R E . L F$ and $R e . L f$, and of the recombinant chromosomes, RE.Lf and Re.LF, among the gametes of the dihybrid.

The inversions $R e$ and $L f$ comprise respectively 8.4 and 5.8 per cent. of the second chromosome and the distance between them represents about 80 per cent. of the chromosome length (da Cunha et al., 1950). These inversions were selected because they are almost as far apart as is possible and, therefore, the probability of detection of rare recombinants, especially in the offspring of the dihybrid males, is maximised.

It is important, at this point, to explain the reasons for seeking recombinants in the offspring of males. The frequencies of recessive lethals in natural populations of $D$. willistoni is studied by means of a series of crosses with laboratory flies having chromosomes marked with both recessive mutations and inversions to prevent recombinations so that flies homozygous for the chromosomes from the natural populations can be produced. Males from nature, or $F_{1}$ from females caught in nature, are crossed, in individual cultures, with flies having $E m$ (emarginate) in one second chromosome and $a b b b w$ in the other. Among the $\mathrm{F}_{1}$ flies from each cross, a single wild-type male, having therefore one second chromosome from the population and one with $a b b b w$, is selected and crossed with a female St Hk $207 a b b b w / b r$. St, Hk and $b r$ stand for the dominants Star and Hook and the recessive broad respectively, all of which are lethal when homozygous. Abbreviated $(a b b)$ and brown $(b w)$ are recessive and viable when homozygous. 207 designates a long pericentric inversion. Among the offspring of this $F_{2}$ cross, five virgin females having $S t H k$ phenotype but non $a b b b w$, being therefore St Hk $207 a b b$ bw/wild are crossed with five males having the same phenotype and genotype. The offspring of this cross is the one analysed to detect the lethal chromosomes from the populations because one of the classes produced, the wild type, will be homozygous for the wild chromosomes from nature. The wild type class must be homozygous genetically and cytologically and will not appear if there is a lethal in the wild chromosome. However, in a few cases, cytological analysis of this class showed that the flies were heterozygous for inversions. Crosses were made very carefully and one strain, out of the 75 " homozygous" strains analysed, was found to be heterozygous for the inversion $L e$ which is located in the mid-region of the left arm of the second chromosome. The inversion could not come from the flies heterozygous for the 207 pericentric inversion. One limit of $L e$ is inside the pericentric and the other is near one of the limits of the pericentric. The only other possibility is that recombination occurs in the chromosomes of the single wild type male $a b b b w /$ wild. If the male is heterozygous $L E / L e$, recombination can give chromosomes $L E$ and $L e$, free from the markers, and these would subsequently be regarded as wild type. Males having one $a b b b w$ and one wild chromosome were analysed cytologically and found to be frequently heterozygous $L E / L e$ as expected.

The strains $R E . L F / R E . L F$ and Re.Lf/Re.Lf, used in the analysis here 
reported, differ also with regard to IIIf, a median inversion which represents about 13 per cent. of the length of the third chromosome.

\section{Results}

Table 1 shows the chromosomal types obtained in the offspring of the male or female, dihybrids, RE.LF/Re.Lf crossed with standard homozygous Re.Lf /Re.Lf flies.

\section{Table 1}

Frequencies of recombinant and non-recombinant chromosomes in the offspring of RE.LF/Re.Le males and females

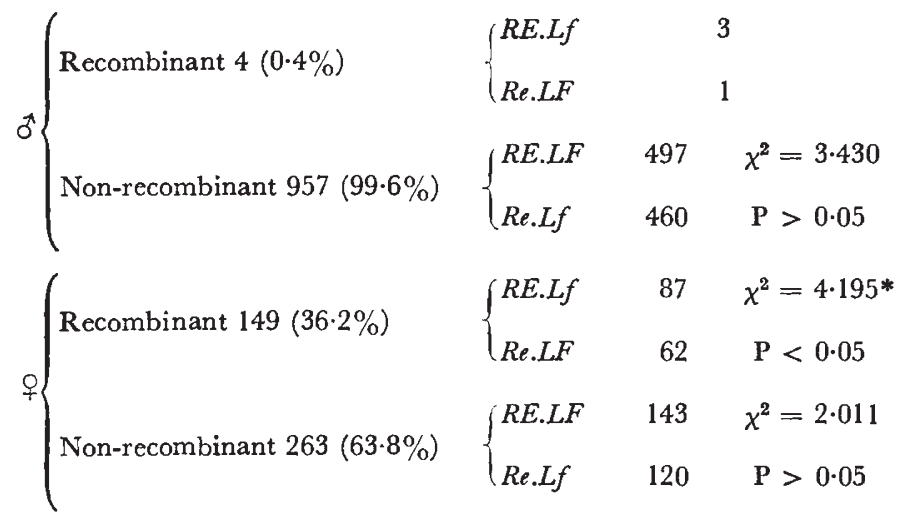

Most of the chromosomes produced by the RE.LF/Re.Lf males are non-recombinant. The two types of non-recombinant chromosomes were obtained in equal frequencies. However, 0.4 per cent. recombinants were obtained among the chromosomes produced by the dihybrid males. This shows that there is crossing-over in $D$. willistoni males.

\section{Table 2}

Frequencies of inversion heterozygotes and homozygotes in back-crosses in which dihybrid males or females were used

Offspring from hybrid males

$\begin{array}{lll}R E / R e & 500 & \chi^{2}=1.583 \\ R e / R e & 461 & \mathbf{P}>0.05 \\ L F / L f & 498 & \chi^{2}=1.275 \\ L f / L f & 463 & \mathbf{P}>0.05\end{array}$

Offspring from hybrid females

$\begin{array}{llc}R E / R e & 230 & \chi^{2}=5.592 * \\ R e / R e & 182 & \mathbf{P}<0.05 \\ & & \\ L F / L f & 205 & \chi^{2}=0.0097 \\ L f / L f & 207 & \mathrm{P}>0.05\end{array}$

Among the chromosomes from dihybrid females, $36 \cdot 2$ per cent. were recombinants and 63.8 per cent. were parental types. The non-recombinant chromosomes were recovered in equal frequencies but the recombinants $R E . L f$ are significantly more common than the alternative type $R e . L F$.

Following back-crossing, larvae heterozygous and homozygous for the two inversions involved should occur in equal frequencies (table 2). Among the offspring of dihybrid males, $R E / R e$ and $L F / L f$ larvae occur more often than the homozygous $R e / R e$ and $L f / L f$ types, but the differences are not statistically significant. 
Among the offspring of the dihybrid females, on the other hand, although the two alternative genotypes $L F / L f$ and $L f / L f$ occur with the same frequencies, the heterozygous $R E / R e$ is significantly more frequent than the alternative homozygous $R e / R e$. These data, obtained in larvae just prior to pupation, suggest differential mortality in favour of the heterozygotes at young larval stages. Interestingly, this difference is found among the $F_{1}$ larvae from hybrid females but not among the offspring from hybrid males. This indicates a possible relationship between differential mortality and the constitution of the egg.

The deviations are consistently in favour of heterozygotes with only one exception in which a deviation is barely evident (table 2).

\section{Discussion}

As Darlington (1958) stated: "A fourth corollary of integration in the genetic system is compromise. Integration means that all components of the genetic system are related to more than one function, all the functions to more than one component. The requirements of the different functions of the same component always differ. They may even be opposed. Hence selection enforces a compromise. The most fundamental compromise is that between high and low crossing-over. Another and related compromise is that between the requirements of hybridity, fertility and stability, a compromise which is solved in entirely different ways in different species." The data here presented illustrate the correctness and the value of Darlington's statement.

Natural populations of $D$. willistoni are highly polymorphic in regard to chromosomal inversions. More than 50 different inversions are known in this species. Twenty-four different inversions are found in the populations of Angra dos Reis from which the chromosomes used in this work were obtained. This polymorphism is adaptive and the inversions protect gene combinations against dissociation by crossing-over (da Gunha, 1956). Heterozygous inversions also inhibit crossing-over in the region of the inverted segments in the Nearctic species of Drosophila. This was well shown by Carson in D. robusta (1953), Dobzhansky and Epling (1948) in D. pseudoobscura, Sturtevant and Beadle (1936) in D. melanogaster, Komai and Takaku $(1940,1942)$ in $D$. virilis, and others. Recombination between homologues would be extremely rare in a large number of natural populations of $D$. willistoni if its inversions had the same suppressive effect on crossing-over outside the inverted segment. D. willistoni has three pairs of chromosomes, two metacentric, one being the $\mathrm{X}$ and one acrocentric. Heterozygotes for two or more inversions of the same chromosome are very common in natural populations of this species. The mean number of heterozygous inversions per female is above three in 27 out of 52 populations studied, close to or above six in 18 populations and near to nine in five populations (da Cunha et al., 1950, 1954, 1959). High frequencies of inversions could not be built at the cost of the almost complete elimination of recombination. The suppression of crossing-over outside the limits of the inversions may be advantageous when there are few inversions, but it restricts the possibility of increase in the number of inversions.

The greatly developed adaptive chromosomal polymorphism in $D$. willistoni is correlated with the high degree of recombination between different inversions. The adaptive compromise between the optimum of 
genotypic integration and the optimum of recombination has been obtained in $D$. willistoni through selection of chromosomal inversions and of recombination between them. This is shown by the data here presented and by preliminary data of Battaglia and Birch (1956). These authors reported 19.7 per cent. recombination between the inversions $L e$ and $L f$ in the left arm of the second chromosome and of 11.7 per cent. between IIIb and IIIj in the third chromosome.

The extent to which inversions suppress crossing over outside their limits is not an intrinsic property of the inversions. It is an adaptive character of the genetic system which may be changed in opposite directions by natural selection. The direction of selection will be determined by environmental conditions and the structure of the population.

Heterozygous inversions of one chromosome may increase crossing-over in others (review in da Cunha, 1955, 1960). This was observed when most of the genotype was tied by inversions. This is not the case in the data here presented. Only a short inversion was present outside the chromosome studied and the amount of recombination found was vastly greater than that observed in the cases of chromosome interaction.

The rate of recombination found in the males, is considerably higher than that obtained in Nearctic species. The recombinants found in the 75 strains made homozygous by crossing with marked strains can be added to this total. The mean of one recombinant in 207 chromosomes, which we found in $D$. willistoni, is of a quite different order of magnitude from the one in 8329 found by Patterson and Suche (1954) in D. melanogaster. The recombinants were found scattered among the offspring of different males and therefore are not clusters of recombinants due to somatic crossing-over.

It is tempting to think that the high frequency of recombinants in the males is correlated with the high frequency of recombinants between inversions in the females. The same group of genes responsible for the high recombination rate in the females may have the increase of recombination in males as a collateral effect. The occurrence in the females of a high rate of recombination between inversions is of obvious importance for the population genetics of $D$. willistoni. Less obvious, owing to its relative rarity, is the importance of the recombination in the males. However, a rate of recombination of one in 200 chromosomes is important if we consider that the mean mutation rate per locus per generation is $1 \times 10^{-5}$ and that one recombination will shuffle thousands of genes. The novelties contributed by the males to the variability of the populations were considered until now to be due only to mutation and to recombination between non-homologous chromosomes. Recombination between homologues in males, even if as low as one in 200, is a new factor the importance of which has to be taken into account.

The occurrence of recombination in males and of a high rate of crossingover between inversions in the females have to be considered carefully in the ordinary methods used to produce homozygous flies. These methods are based on the assumptions that no crossing-over occurs in males and that heterozygous inversions suppress crossing-over outside the limits of the inversions. The data reported show that the two assumptions are not always true.

The genetic systems of closely related species may differ and comparative studies may help in the understanding of the adaptive meaning of the differences and of the evolution of the systems as an integrated whole. 


\section{SUMmary}

1. In Nearctic species of Drosophila there is no crossing-over in the males. In females heterozygous for inversions, recombinants produced by crossingover within the inversions are eliminated and recombination outside the inverted segments is inhibited.

2. The genetic system of Drosophila willistoni differs from those of the Nearctic species.

3. D. willistoni is a Neotropical species characterised by populations highly polymorphic for chromosomal inversions. In females a high rate of recombination occurs between different heterozygous inversions on the same chromosome.

4. Crossing-over occurs in males. The rate though low, about one recombinant per 200 chromosomes, is much higher than in $D$. melanogaster with about one recombinant in 8300 chromosomes.

5. The suppression of recombination within inverted chromosomal segments, the occurrence of crossing-over between heterozygous inversions and the development of adaptive chromosomal polymorphism are related.

Acknowledgments. - This work is part of projects in progress with financial aid from the US-AEC(CONTRACT AT(30-1)-2733, THE ROCKEFELLER FOUNDATION, FAPESP, CNPq, CAPES and CNEN. One of the authors, Z. M. França, held a fellowship from the FAPESP. The authors are very grateful to these Institutions and to Professor C. Pavan and Misses Therezinha de Moraes Ungaretti and Ilze Lamber Jorge who helped in many ways.

\section{REFERENCES}

BAtTAglia, B., AND BIRCH. 1956. Crossing-over in Drosokhila willistoni. Nature, 178, 1005. CARSON, H. L. 1953. The effects of inversions on crossing-over in Drosophila robusta. Genetics, $38,168-186$.

DA Cunha, A. B. 1955. Chromosomal polymorphism in the Diptera. Advanc. Genet., 7, 93-138.

DA CUNHA, A. B. 1956. Contribuição ao estudo da adaptação das populações de Drosophila a diferentes levedos, Bol. Fac. Fill. Ciên. e Letras Univ. S. Paulo-Biologia Geral., 10, 1-56.

DA CunHA, A. B. 1960. Chromosomal variation and adaptation in insects. Ann. Rev. Entomol., 5, 85-110.

DA CUNHA, A. B., BURLA, H., AND DOBzhansky, T. 1950. Adaptive chromosomal polymorphism in Drosophila willistoni. Evolution, 4, 212-235.

DA GUNHA, A. B., AND DOBzHANSKY, T. 1954. A further study of chromosomal polymorphism in Drosophila willistoni in its relation to the environment. Evolution, 8, 119-134.

DA CUNHA, A. B., DOBZHANSKy, T., PAVlovsky, O., AND SPASSKY, B. 1959. Genetics of natural populations. XXVIII. Supplementary data on the chromosomal polymorphism in Drosophila willistoni in its relation to the environment. Evolution, 13, 389-404.

DARLINGton, C. D. 1958. Evolution of Genetic Systems (2nd ed.). Oliver and Boyd. Edinburgh and London.

DOBZHANSKY, T., AND EPLING, c. 1948. The suppression of crossing over in inversion heterozygotes of Drosophila pseudoobscura. Proc. Nat. Acad. Sci., Wash., 34, 137-141.

KomaI, T., AND TAKaKU, T. 1940. Two independent inversions in the X-chromosome of Drosophila virilis and their effects on crossing-over and disjunction. Cytologia, 11, 245-260.

KOMAI, T., AND TAKAKU, T. 1942. On the effect of the X-chromosome inversions on crossing-over in Drosophila virilis. Cytologia, 12, 357-365.

MORGan, T. H. 1914. No crossing-over in the male of Drosophila of genes in the second and third pairs of chromosomes. Biol. Bull., 26, 195-204.

PATTERson, J. T., AND SUChe, M. L. 1934. Crossing over induced by X-rays in Drosophila males. Genetics, 19, 223-236.

STURTEVANT, A. H., AND BeAdLE, G. w. 1936. The relations of inversions in the X-chromosome of Drosophila melanogaster to crossing-over and disjunction. Genetics, 21, 554-604. 